\title{
Analysis of PEA photopolymers at zero spatial frequency limit
}

\author{
Sergi Gallego ${ }^{* a, b}$, Andrés Márquez ${ }^{\mathrm{a}, \mathrm{b}}$, Marina Riquelme ${ }^{\mathrm{b},}$, Cristian Neipp ${ }^{\mathrm{a}, \mathrm{b}}$, Manuel Ortuño ${ }^{\mathrm{a}, \mathrm{b}}$, \\ Augusto Beléndez ${ }^{\mathrm{a}, \mathrm{b}}$, Inmaculada Pascual ${ }^{\mathrm{b}, \mathrm{c}}$ \\ ${ }^{a}$ Dept. de Física, Ing. de Sistemas y Teoría de la Señal, Universidad de Alicante, P.O. Box 99, E-

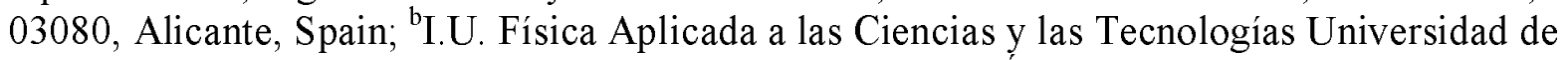 \\ Alicante, P.O. Box 99, E-03080, Alicante, Spain; ' Dept. de Óptica, Farmacología y Anatomía, \\ Universidad de Alicante, P.O. Box 99, E-03080, Alicante, Spain
}

\begin{abstract}
The PEA photopolymer is composed of dipentaerythritol penta/hexa-acrylate as monomer and binder, $\mathrm{N}$-vinyl pirrolidone as crosslinker, ethyl eosin as dye and N-methyl diethanolamine as radical generator. This photopolymer is suitable to work with dispersed liquid crystal molecules in dynamic holographic and diffractive applications. In order to characterize these materials we have analyzed the behaviour of different compositions at zero spatial frequency limit. This method is based on an interferometer that has been successfully applied in the phase-shift versus applied voltage characterization of liquid-crystal displays, in addition to that it has been applied to characterize PVA/AA and PVA/NaAO photopolymers. In PEA case there is no shrinkage since the photopolymer is coverplated. Samples have a glass substrate as the cover plate. In our analysis we have studied the importance of the monomer, crosslinker and crystal liquid molecules concentrations, in the phase shift produced in the layer during photopolymerization process.
\end{abstract}

Keywords: Holographic data storage, Holographic recording materials, Diffractive optics, liquid crystals

\section{INTRODUCTION}

Photopolymers have been investigated so far for holographic/photonic applications such as diffractive elements, datastorage displays, optical waveguides, etc [1-7]. There are many types of photopolymers that may be differentiated by the type of binder, since this component determines to a great extent the choice of monomer, dye and initiator used in the photopolymer. Analyzing the behavior of a photopolymer as an optic storage medium is a complicated task. Normally, these materials are used in holographic applications, where high values of spatial frequencies are recorded. In this range of frequencies many processes are involved in the hologram formation, such as species diffusion, nonlocalpolymerization (due to the finite size of polymer chains), and shrinkage or swelling (volume changes) [8-10]. In this sense, the variations in the estimation of the monomer diffusion affect significantly the values obtained for other material parameters, making it difficult to calculate them separately. In particular, it is interesting to analyze the material behavior at very low spatial frequencies, since at these frequencies monomer diffusion does not play an important role and even disappears at the zero spatial frequency limit. This paper is the continuation of a previous works, where some compositions of photopolymers were studied at the zero frequency limit using a transmission and reflection interferometric setup [10-12].

In the case analyzed in this work, PEA photopolymers with and without dispersed liquid crystal molecules. This material is liable to contain liquid crystal particles, thus dynamic holograms or diffractive elements can be manufactured. The formation of switchable holographic gratings from polymer dispersed liquid crystals (H-PDLCs) allows for the development of switchable transmissive and reflective diffractive optics. To overcome some limitations of photopolymers and Liquid Crystal Displays, there has been a fascinating marriage of two polymer-related technologies over the past few years: photopolymer holography and polymer-dispersed liquid crystals, H-PDLC [1, 13]. Local differences in photopolymerization rates induce phase separation of discrete LC domains to occur periodically commensurate with the period of the interference pattern. When exposing such a mixture to the optical field of two or more interfering laser beams, photopolymerization occurs more rapidly in bright than in dark regions of the interference pattern. This leads to diffusion of liquid crystalline molecules into dark regions and of monomers to bright regions. The applications, new H-PDLC compositions and optimization studies are presented in several papers [13-19]. In general the

Optical Modelling and Design II, edited by Frank Wyrowski, John T. Sheridan, Jani Tervo, Youri Meuret, Proc. of SPIE Vol. 8429, 842925 - (c) 2012 SPIE · CCC code: 0277-786X/12/\$18 - doi: 10.1117/12.921789 
samples are between two glasses. This cover-plating can provide mechanical support and alter surface tension (energy) effects so as to change the profile of the surface relief grating formed during exposure [8]. In general the phase shift between the exposed and non-exposed areas in the transmission interferometric case is due to two different effects: monomer polymerization in the exposed zones and thickness variations when polymerization occurs. With coverplated samples we cannot use a reflection interferometric setup to analyze the thickness variation during exposition at the zero spatial frequency limit. In this work we analyzed different chemical composition of PEA photopolymers at zero spatial frequency limit, we also studied the effects of the temperature in the material behavior and the influence of the dispersed liquid crystal molecules in the polymerization process.

Only we can measure the results in transmission, where both refractive index and volume variations have influence. The phase shift measured in the transmission case $\left(\Delta \Phi_{t}\right)$ can be described as follows:

$$
\Delta \Phi_{t}=\frac{2 \pi}{\lambda} d(t) \cdot \Delta n \cos \alpha^{\prime}-\Delta \Phi_{S h r}
$$

where $d(\mathrm{t})$ is the new "solid" layer thickness [11-12], $\alpha^{\prime}$ is the angle inside the material and $\Delta \Phi_{S h r}$ is the phase shift due to the shrinkage. For cover-plated photopolymers we can consider that the volume between the two glass substrates remains constant.

The equation can be simplified for small incident angles $\left(\cos \alpha^{\prime}=\cos \alpha=1\right)$ and used to calculate the refractive index variation. In the case, for the whole layer the refractive index variation $\left(\Delta n_{w}\right)$ can be calculated as follows:

$$
\Delta n_{w}=\frac{\lambda \Delta \Phi_{t} \cos \alpha^{\prime}}{2 \pi d_{0}}
$$

Along the polymerization process, the holes are expulsed out of the "solid" layer and so its density increases [12].

$$
\phi^{(m)}+\phi^{(p)}+\phi^{(h)}+\phi^{(b)}=1
$$

where $\phi^{(m)}, \phi^{(p)}, \phi^{(h)}$ and $\phi^{(b)}$ are the volume fractions of the monomer, the polymer, the holes and the background (PVA, triethanolamine and dye) respectively. While the collapse of holes will result in a reduction in the overall volume, the total volume fraction is by definition conserved. We can assume that $\phi^{(b)}$ remain constant during the exposition and polymer and holes (on the surface) begin to appear when the monomer starts to polymerize. Therefore we can assume that

$$
\phi^{(m)}+\phi^{(p)}+\phi^{(h)}=\phi_{0}^{(m)}
$$

Where, $\phi_{0}^{(m)}$ is the initial volume fraction of the monomer.

At zero spatial frequency limit, when diffusion does not take place, the decreasing of the monomer concentration depends on the recording intensity, $I$, the dependency of the polymerization and intensity, $\gamma$, initial monomer concentration and the polymerization reaction constant, $K_{R}$.

$$
\phi^{(m)}(t)=\phi_{0}^{(m)} e^{-I^{\gamma} K_{R} t}
$$

Following the same steps presented in [11-12] we can obtain the polymerization rate as a function of the experimental values of the phase shift, $P S$, during recording.

$$
\ln \left(1-\frac{P S(t)}{P S_{\infty}}\right)=-I^{\gamma} K_{R} t=-F_{R} t
$$




\section{EXPERIMENTAL SET-UP}

This photopolymer is composed of dipentaerythritol penta/hexa-acrylate (DPHPA) as monomer and binder, N-vinyl pirrolidone (NVP) as crosslinker, ethyl eosin (YE) as dye and N-methyl diethanolamine (NMDETA) as radical generator. These components were mixed under red light where the material is not sensitive and under a temperature of $40{ }^{\circ} \mathrm{C}$ to solve properly all the components, specially the crystal liquid molecules. The solution $(100 \mathrm{~mL})$ was sonicated in an ultrasonic bath, heated, mixed with a magnetic mixer and deposited between two glass plates $2 \mathrm{~mm}$ thick. The HPDLCs layers have thickness around $10 \mu \mathrm{m}$ in order to apply the electric field and to align the crystal liquid molecules, thus obtain the switchable holographic gratings. In the samples analyzed in this work we have used spacing of $106 \mu \mathrm{m}$ to increase the phase shift and see properly the different behaviours reducing the error in our calculations. The focus of this goal is the characterization of the chemical composition, we don't make switchable gratings. In Table 1 we described the composition of different layers analyzed at zero spatial frequencies. These compositions are designed to keep constant the liquid volume. In the first three compositions we analyze the influence of crosslinker concentration; in the last four we analyze the influence of the liquid crystal in the polymerization process.

Table 1. Chemical compositions analyzed.

\begin{tabular}{|l|c|c|c|c|c|c|c|}
\hline & C1 & C2 & C3 & C4 & C5 & C6 & C7 \\
\hline DPHPA & $2 \mathrm{~g}$ & $1.9 \mathrm{~g}$ & $1.7 \mathrm{~g}$ & $1.4 \mathrm{~g}$ & $1.5 \mathrm{~g}$ & $1.6 \mathrm{~g}$ & $1.8 \mathrm{~g}$ \\
\hline NMDETA & $400 \mu \mathrm{L}$ & $400 \mu \mathrm{L}$ & $400 \mu \mathrm{L}$ & $400 \mu \mathrm{L}$ & $400 \mu \mathrm{L}$ & $400 \mu \mathrm{L}$ & $400 \mu \mathrm{L}$ \\
\hline NVP & $100 \mu \mathrm{L}$ & $200 \mu \mathrm{L}$ & $400 \mu \mathrm{L}$ & $100 \mu \mathrm{L}$ & $100 \mu \mathrm{L}$ & $100 \mu \mathrm{L}$ & $100 \mu \mathrm{L}$ \\
\hline YEt & $0.001 \mathrm{~g}$ & $0.001 \mathrm{~g}$ & $0.001 \mathrm{~g}$ & $0.001 \mathrm{~g}$ & $0.001 \mathrm{~g}$ & $0.001 \mathrm{~g}$ & $0.001 \mathrm{~g}$ \\
\hline LC BL087 & & & & $600 \mu \mathrm{L}$ & $500 \mu \mathrm{L}$ & $400 \mu \mathrm{L}$ & $200 \mu \mathrm{L}$ \\
\hline
\end{tabular}

In this Section we focus on the description of the combined setup to obtain real time interferometric measurements while the holographic material is being exposed. We used the combined setup with the interferometer in transmission. In Fig. 1 we show the experimental setup in transmission geometry to measure the phase-shift as a function of the exposure energy. The setup has two arms with an angular separation of $14^{\circ}$, one to expose the recording material, whereas the second arm is the interferometer used to measure, in real-time, the phase-shift. The recording material is perpendicularly oriented with respect to the interferometer axis in order to ease the analysis of the interferometric results: at an oblique incidence we should take into account both the Fresnel coefficients at the interface and the increase of distance in the propagation across the layer.

In the first arm, the exposure beam provided by a solid-state $\mathrm{Nd}-\mathrm{YVO}_{4}$ Verdi laser with a wavelength of $532 \mathrm{~nm}$ (at this wavelength the dye presents the maximum absorption) is expanded and collimated using a spatial filter and a lens, obtaining a beam with $1.5 \mathrm{~cm}$ of radius. A wave plate and a neutral filter (attenuator) are added before the spatial filter to control the orientation and the intensity of the linearly polarized beam produced by the laser $\mathrm{Nd}-\mathrm{YVO}_{4} . \mathrm{A}^{2}$ polarizer $(\mathrm{P})$, with its transmission axis oriented along the vertical of the lab, is introduced to produce a beam with TE polarization incident onto the recording material. This incident beam forms an angle of 14 degrees with respect to the photopolymer layer. We adjust the laser power so that the exposure intensity that impinges on the layer is $2.5 \mathrm{~mW} / \mathrm{cm}^{2}$ (this is the value corrected from the Fresnel coefficient at the air-photopolymer interface at an incidence of 14 degrees for TE polarization). A half-opened diaphragm is used to leave an unexposed area in the photopolymer layer.

In the interferometric arm, to generate the interferences pattern we use a He-Ne laser, since the photopolymer does not present any absorption at $633 \mathrm{~nm}$. We have implemented a Young's fringes based two beams interferometer. This interferometer has been successfully applied in the phase-shift characterization of liquid crystal displays (LCDs) [20]. It shows a good precision, and due to its quasi-common path architecture is a robust setup, less sensible to changing environmental conditions and simpler to construct than Mach-Zehnder type interferometers. We use a grating with a spatial frequency of 4 lines $/ \mathrm{mm}$ to generate a series of diffracted orders from the unexpanded He-Ne beam; we block all the orders except -1 and +1 . One of the two orders impinges on the exposed zone (illuminated by the $\mathrm{Nd}_{-} \mathrm{YVO}_{4}$ laser) and the other one impinges on the non-exposed zone. The distance between the two orders is about $1 \mathrm{~cm}$, so as to eliminate the influence of the monomer diffusion in the polymerization process. Once the two orders have propagated throughout the photopolymer, a lens is used to make them interfere. A microscope objective is used to amplify the 
interference pattern onto a CCD camera. This pattern is captured in real-time as a function of exposure at specific time intervals.

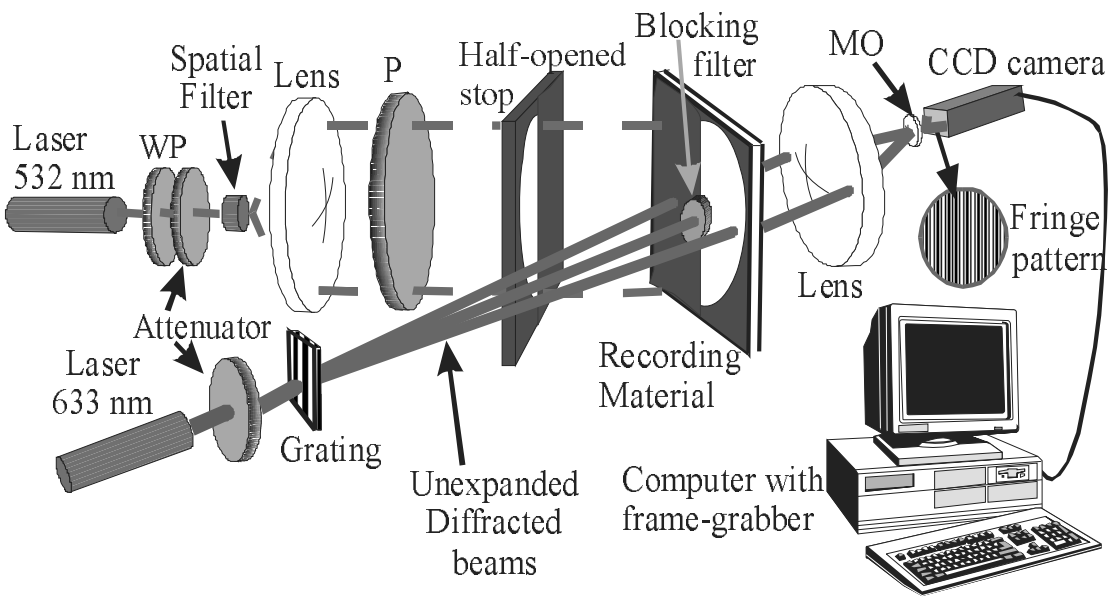

Fig. 1. Experimental setup. The recording material is exposed with the green laser beam $(\lambda=532 \mathrm{~nm})$ and the phase-shift is measured with the red beam $(\lambda=633 \mathrm{~nm})$. $\mathrm{P}$ is polarizer, WP is wave plate, MO is microscope objective.

Therefore, with this setup it is possible to plot the fringes moving as a function of the exposure time during the polymerization process, and without the influence of monomer diffusion. In this sense, we have verified that before and once we stop the exposition, the shift in the interference fringes freezes. This indicates that the measurements taken in this setup are not affected by diffusion processes.

\section{RESULTS AND DISCUSSION}

In this section we present the experimental data obtained for different material compositions, and for different cooling times. We focus our attention on the best experimental procedure and chemical composition to achieve stable and high phase shift between exposed and non exposed areas. Following this idea, we study the influence of the cooling time, the crosslinker concentration, and the quantity of crystal liquid. Then we can find which is the optimum procedure and chemical composition.

In the case of our PEA photopolymer we have to heat the solution in order to solve all the components. Therefore when the temperature is cooling down there are some problems to keep the mixture solved and the polymer rates are modified. This is an important drawn back in the way of the possible commercialization of this type of photopolymers. In our analysis of the cooling time we have chosen 5 different times. And the response at zero spatial frequency limits is depicted in Figure 2. As can be seen when the layer is exposed without cooling time, the material behavior present two different slopes, before $25 \mathrm{~s}$ and after $25 \mathrm{~s}$. Layers cooled down around 1 and 5 minutes present very similar behavior and when the cooling time is longer than 10 minutes. Therefore we assume that the diffractive image should be recorded in these materials between 1 and 7 minutes. Other possibility is to use the oven the sample to keep all the properties constant.

For instance, when crosslinker monomer is added, we expect that the polymerization rates increase drastically [21]. Nevertheless in the cases studied the compositions with crosslinker have low quantity of monomer in order to keep constant the polymer solution volume. In Figure 2 we show the phase shift between exposed and non-exposed zones for three different crosslinker compositions. We can see the clear increase of the phase shift due to crosslinker. Nevertheless the composition with the highest crosslinker presents different slopes, and the final phase shift is more or less the same of the compositions with 200 and $100 \mathrm{ml}$. Thus we consider the composition with $100 \mathrm{ml}$ of crosslinker the optimum, furthermore this presents more regular behavior. 


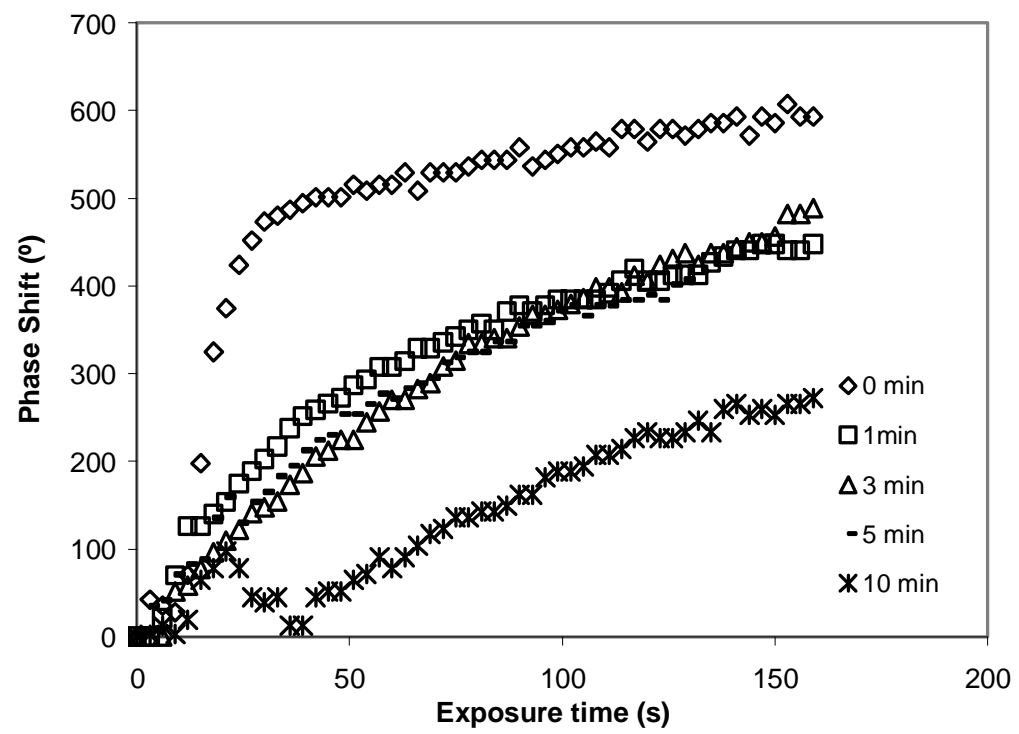

Fig. 1. Behavior of Photopolymer $\mathrm{C} 1$ at the zero spatial frequency limit in transmission for different cooling times.

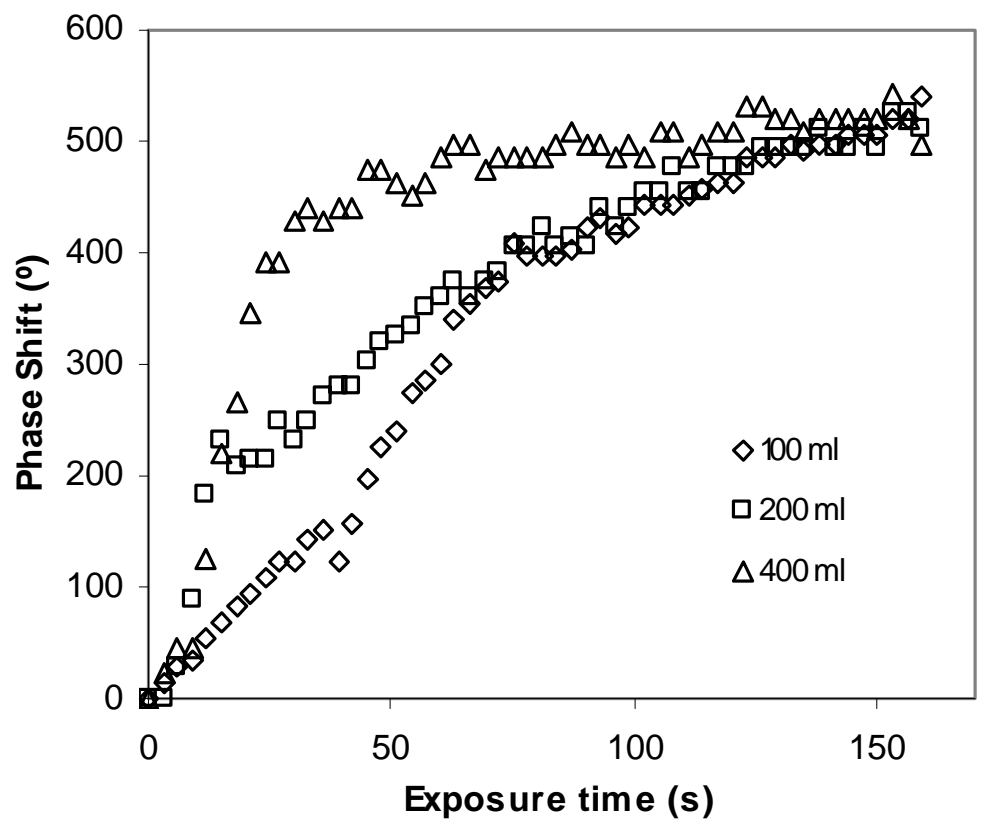

Fig. 2. Behavior of Photopolymers C1, C2 and C3 at the zero spatial frequency limit in transmission for 5 minutes of cooling times.

To obtain the magnitude of the velocity of the chemical reaction, the polymerization rate, $F_{R}$, we can use Eq. (6). In Fig. 3 we have depicted the results for composition $\mathrm{C} 1$ obtained by the fitting based on Eq. (6). The correlation value of the fittings indicates the good agreement between theory and experiments. 


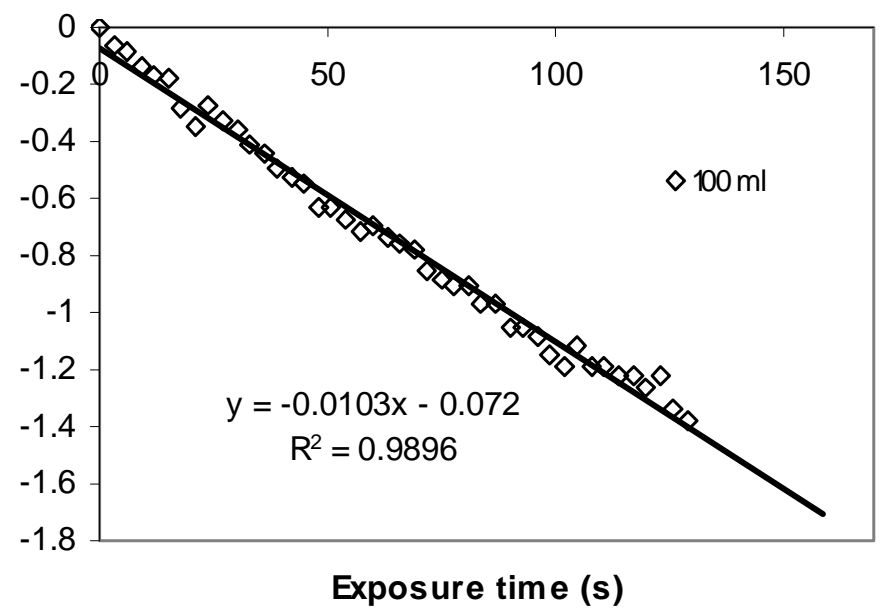

Fig. 3. Fitting of the polymerization rate for polymer $\mathrm{C} 1$ at the zero spatial frequency limit for 5 minutes of cooling time.

To study the influence of the crystal liquid molecules in the photopolymer kinetics without crosslinker we have exposed under green light 5 different compositions ( $\mathrm{C} 1, \mathrm{C} 4, \mathrm{C} 5, \mathrm{C} 6$ and $\mathrm{C} 7)$. At zero spatial frequency limit the phase separation of the liquid crystal don't exist, therefore it is difficult to compare with the holographic case. Nevertheless we can extract the idea of the influence of the liquid crystal on the photopolymerization process. It is important to note that we have modified the monomer concentration to keep the solution volume constant. As can be seen the compositions with $600 \mathrm{ml}$ and 500 present identical behavior, and the reduction of the final phase shift is due to the presence of liquid crystal molecules is weak but affect. The phase shift between exposed and non exposed zones is around $600^{\circ}$ for composition $\mathrm{C} 1$ and less of $300^{\circ}$ for composition $\mathrm{C} 7$ and $\mathrm{C} 1$ have $2 \mathrm{~g}$ of monomer and $\mathrm{C} 71.4 \mathrm{~g}$. It is important to remark that only the presence of Crystal liquid molecules at zero spatial frequency, without phase separation, makes that the variations in the average refractive index smaller. There is a non-variable component in the solution.

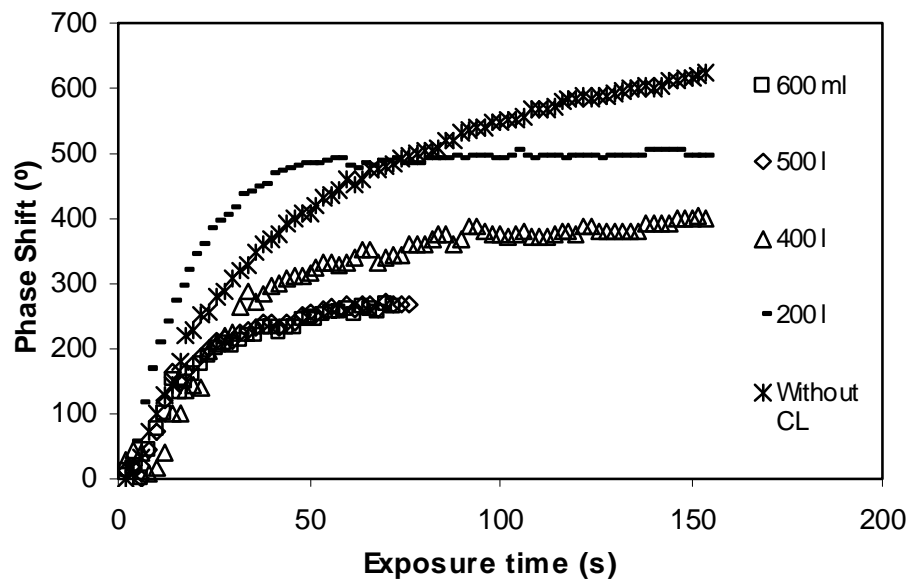

Fig. 4. Behavior of Photopolymers C1, C4, C5, C6 and C7 at the zero spatial frequency limit in transmission for 5 minutes of cooling time. 


\section{CONCLUSIONS}

We have studied different chemical compositions of PEA photopolymer at zero spatial frequency limit. We have show how large cooling times affect to the material behaviour. Thus we have proposed times between 1 and 7 minutes to use the material as optic recording material. We also have studied the influence of crosslinker concentration in the polymerization and phase shift. On the last place we have studied the effects of the crystal liquid in the solution, we have conclude that the presence of liquid crystal reduces the phase shift between exposed and non-exposed areas, but we must determine with additional experiments in which way affects to the polymerization rate and the average refractive index of the layer.

\section{Acknowledgments}

This work was supported by the Ministerio de Ciencia e Innovación of Spain under projects FIS2011-29803-C02-01 and FIS2011-29803-C02-02 and by the Generalitat Valenciana of Spain under project PROMETEO/2011/021

\section{REFERENCES}

[1] N. Suzuki, Y. Tomita, K. Ohmori, M. Hidaka, and K. Chikama, "Highly transparent $\mathrm{ZrO}_{2}$ nanoparticle-dispersed acrylate photopolymers for volume holographic recording," Opt. Express 14, 12712-12719 (2006).

[2] R. A. Lessard and G. Manivannan, "Holographic recording materials: an overview," Proc. SPIE 2405, 2-23(1995).

[3] T. J. Trout, J. J. Schmieg, W. J. Gambogi, and A. M. Weber, "Optical photopolymers: design and applications,"Adv. Mater. 10, 1219-1224(1998).

[4] V. A. Barachevskii, "Photopolymerizable recording media for three-dimensional holographic optical memory," High. Energy Chem. 40, 131-141(2006).

[5] W. J. Gambogi, A. M. Weber, and T. J. Trout, "Advances and applications of DuPont holographic photopolymers, SPIE 2043, 2-13(1993).

[6] H.-Y. S. Li and D. Psaltis, "Three-dimensional holographic disks," Appl. Opt. 33, 3764-3774(1994).

[7] S. Orlic, S. Ulm, and H. J. Eichler, "3D bit-oriented optical storage in photopolymers," J. Opt. A 3, 72-81(2001).

[8] C. E. Close, M. R. Gleeson and J. T. Sheridan, "Monomer Diffusion Rates in Photopolymer Material: Part I: Low Spatial Frequency Holographic Gratings" JOSA B 28, 658-666, (2011).

[9] I. Naydenova, E. Mihaylova, S. Martin, and V. Toal, "Holographic patterning of acrylamide-based photopolymer surface," Opt. Express 13, 4878-4889 (2005).

[10] S. Gallego, A. Márquez, M. Ortuño, J. Francés, S. Marini, A. Beléndez, and I. Pascual, "Surface relief model for photopolymers without cover plating," Opt. Express 19, 10896-10906 (2011).

[11]S. Gallego, A. Márquez, D. Méndez, C. Neipp, M. Ortuño, M. L. Alvarez, E. Fernandez, A. Beléndez, and I. Pascual, "Real-time interferometric characterization of a polyvinyl alcohol based photopolymer at the zero spatial frequency limit," Appl. Opt. 46(30), 7506-7512 (2007).

[12] S. Gallego, A. Márquez, M. Ortuño, S. Marini, J. Francés "High environmental compatibility photopolymers compared to PVA/AA based materials at zero spatial frequency limit" Opt. Materials 33, 531-537. (2010).

[13] S. Harbour , J.V. Kelly , T. Galstian , J.T. Sheridan, Optical birefringence and anisotropic scattering in acrylate based holographic polymer dispersed liquid crystals Optics Communications 278 (2007) 28-33

[14] K. R. Sun, Y. S. Kang, B. K. Kim "Transflective multiplexing of holographic polymer dispersed liquid crystal using Si additives" Polymer letters (2010)

[15] T. J. Bunning, L. V. Natarajan, V. P. Tondiglia, and R. L. Sutherland "Holographic polymer-dispersed liquid crystals (H-PDLCS)" Annu. Rev. Mater. Sci. 30:83-115, (2000).

[16] R. L. Sutherland, V. P. Tondiglia, L. V. Natarajan, and T. J. Bunning, "Phenomenological model of anisotropic volume hologram formation in liquid- crystal-photopolymer mixtures," J. Appl. Phys. 96, 951-965 (2004).

[17]K. Tanaka, K. Kato, S. Tsuru, and S. Sakai, "Holographically formed liquid-crystal/polymer device for reflective color display," J. Soc. Inf. Disp. 2, 37-40 (1994).

[18]M. A. Ellabban, M. Fally, H. Uršič, and I. Drevenšek-Olenik, "Holographic scattering in photopolymer-dispersed liquid crystals" Appl. Phys. Lett. 87, 151101 (2005).

[19]H. Gao , K. Gu, Z. Zhou, Y. Jiang, D. Gong "Diffraction behavior of an azo-dye-doped nematic liquid crystal without applied electric field“ Current Applied Physics 8 31-35 (2008). 
[20] A. Bergeron, J. Gauvin, F. Gagnon, D. Gingras, H. H. Arsenault and M. Doucet, "Phase calibration and applications of a liquid-crystal spatial light modulator", Appl. Opt. 34, 5133-5139 (1995).

[21]Cristian Neipp, Sergi Gallego, Manuel Ortuño, Andrés Márquez, Augusto Beléndez, Inmaculada Pascual "Characterization of a PVA/acrylamide photopolymer. Influence of a cross-linking monomer in the final characteristics of the hologram" Optics Communications, 224, 27-34 (2003). 